\title{
INFORMAÇÕES DOS ADOLESCENTES SOBRE MÉTODOS ANTICONCEPCIONAIS
}

\author{
Alzira Maria d'Ávila Nery Guimarães ${ }^{1}$ \\ Maria Jésia Vieira ${ }^{2}$ \\ José Arnaldo Palmeira ${ }^{3}$
}

Guimarães AMDN, Vieira MJ, Palmeira JA. Informações dos adolescentes sobre métodos anticoncepcionais. Rev Latino-am Enfermagem 2003 maio-junho; 11(3):293-8.

Realizou-se estudo de corte transversal, com o objetivo de identificar, em escolares do ensino médio, informações relativas aos métodos anticoncepcionais. A amostra compreendeu 816 adolescentes de ambos os sexos de escolas públicas de Aracaju, Sergipe. Em relação a essas informações, foram investigados também aspectos demográficos, pedagógicos, comportamentais e reprodutivos. Verificou-se que $59 \%$ dos adolescentes possuíam vida sexual ativa e $57,7 \%$ afirmaram não receber informações sobre métodos anticoncepcionais nas escolas. As fontes de informações, revistas, livros e jornais, alcançaram o percentual de $28 \%$ e o condon masculino (84,5\%) foi o método mais conhecido. A análise possibilitou identificar a necessidade de maior discussão na escola e a inserção dos pais nesse processo.

DESCRITORES: adolescência, métodos anticoncepcionais, educação sexual

\section{TEENAGERS" INFORMATION ABOUT ANTICONCEPTIVE METHODS}

A transversal study was realized in order to identify information about anticonceptive methods in high school students. The sample included 816 adolescents of both genders from public schools in Aracaju, Sergipe. With respect to this information, demographic, pedagogical, behavioral and reproductive aspects were also analyzed. It was verified that $59.0 \%$ of the teenagers have an active sexual life and $57.7 \%$ affirmed that they do not receive information about anticonceptive methods in school. Magazines, books and newspapers obtained $28 \%$ as sources of information and the male condom (84.5\%) was the best known method. The analysis made it possible to identify the need for further discussion in school and the insertion of the parents in this process.

DESCRIPTORS: adolescence, anticonceptive methods, sexual education

\section{INFORMACIONES LOS ADOLESCENTES SOBRE LOS} MÉTODOS ANTICONCEPTIVOS

Se realizó un estudio de corte transversal, con el objetivo de identificar en estudiantes de enseñanza media, informaciones relacionadas con los métodos anticonceptivos. La muestra comprendió 816 adolescentes de ambos sexos de escuelas públicas de Aracaju Estado de Sergipe. Se averiguaron aspectos demográficos, pedagógicos, comportamentales y reproductivos. Se encontró que 59\% de los adolescentes tienen vida sexual activa y 57,7\% afirmaron no haber recibido informaciones sobre los métodos anticonceptivos en las escuelas. Las fuentes de información, revistas, libros y periódicos alcanzaron un porcentaje de $28 \%$ y el condón masculino $84,5 \%$, fue el método más conocido. El análisis hizo posible identificar la necesidad de mayor discusión en la escuela y la inclusión de los padres en este proceso.

DESCRIPTORES: adolescencia, métodos anticonceptivos, educación

\footnotetext{
${ }^{1}$ Enfermeira, Professor Mestre Assistente, endereço: Rua: Alameda B, 303 - Ap. 702 - Cond. Tropical Vilage - Jardins - 49025-100 - Aracajú Sergipe - Brasil; ${ }^{2}$ Professor Adjunto Doutor; ${ }^{3}$ Médico, Professor Titular. Universidade Federal de Sergipe
} 
INTRODUÇÃO

$\boldsymbol{O}$ homem, como ser político, relaciona-se com o meio, transformando-o e, conseqüentemente, participa do processo histórico.

A anticoncepção faz parte da história do homem e refere-se, mais especificamente, à prevenção temporária da gravidez, o que dá origem à terminologia utilizada em relação aos métodos anticoncepcionais e seu uso ${ }^{(1)}$.

A utilização de qualquer método anticonceptivo constitui produto de decisão consciente das relações existentes entre os vários subprocessos experimentados pelos indivíduos em sua vida e, mais especificamente, num relacionamento sexual.

A forma que o indivíduo vivencia esse processo é fortemente influenciada por seu conhecimento sobre prática sexual, gravidez e risco de engravidar, que também é influenciada pelo conhecimento sobre métodos anticoncepcionais ${ }^{(2)}$.

Se a anticoncepção não é uma tarefa fácil para o adulto, torna-se ainda mais complexa para $\mathrm{o}$ adolescente.

A atenção ao grupo de adolescentes passa a ser cada vez mais reconhecida como necessária, devido, principalmente, à sua composição numérica, à freqüência cada vez maior da gravidez na adolescência, dos acidentes, da violência, do uso de tabaco, álcool, drogas, inalantes, além dos problemas de saúde mental( ${ }^{(3)}$.

A magnitude numérica dos adolescentes traduzse pela existência no Brasil de 35,5 milhões de adolescentes, o que corresponde a $23,4 \%$ da população brasileira. Desse quantitativo, $46,6 \%$ são de sexo feminino e $50,4 \%$ do masculino ${ }^{(4)}$.

Os números dispensam justificativas para a importância que deve merecer a saúde dessa população. Deve ser considerado, entretanto, que nem todos os adolescentes sofrem dos mesmos problemas e devem ser tratados do mesmo modo, sendo fundamental o respeito à individualidade, principalmente por sermos um país de dimensões continentais, de muitas culturas e casos flagrantes de desigualdade social ${ }^{(5)}$.

Em relação à anticoncepção, dados do Ministério da Saúde apontam que $55 \%$ das adolescentes solteiras e sexualmente ativas, no Brasil, nunca haviam usado nenhum método anticoncepcional, número que se eleva para $79 \%$ nas áreas rurais ${ }^{(6)}$.

As adolescentes engravidam na sua grande maioria sem planejamento, por falta de informação, difícil acesso aos serviços de saúde e desconhecimento sobre métodos anticoncepcionais, além da busca afetiva, de um objeto de amor ou somente experimentação sexual.

Educadores, profissionais de saúde e pais, participantes ativos da formação dos adolescentes, com freqüência, não têm consciência ou sensibilidade quanto ao problema desse grupo populacional: devido à falta de informação ou, simplesmente, ao constrangimento em discutir temas ligados à sexualidade, poupando 0 adolescente de usufruir do direito de escolha, com base em informações contextualizadas, de acordo com suas características de vida.

Assim, este estudo justifica-se pela necessidade de mostrar de forma mais abrangente a real dimensão do assunto, oportunizando escolas e serviços de saúde a prestarem uma assistência de qualidade, voltada para o adolescente.

A partir dessa problemática, objetivamos, com este estudo, analisar as informações dos adolescentes escolares sobre métodos anticoncepcionais.

\section{MÉTODOS}

O estudo descritivo foi o método utilizado para a realização desta pesquisa.

O trabalho teve como base territorial a cidade de Aracaju, capital do Estado de Sergipe, com população de 428194 habitantes, distribuídos em sua totalidade na área urbana, concentrando $26,39 \%$ da população de todo o Estado.

Em relação ao quantitativo de adolescentes, o município tinha no ano 2000, 99099 indivíduos, correspondendo a $21 \%$ de toda sua população ${ }^{(7)}$.

De acordo com o Serviço de Informação e Estatística da Secretaria de Estado de Educação de Desporto e Lazer de Sergipe ${ }^{(8)}$, existiam em Aracaju 54 estabelecimentos escolares de ensino fundamental e médio. Estavam matriculados na rede pública no ensino médio 20012 alunos.

A escolha das escolas públicas do ensino médio de Aracaju foi realizada através de amostragem por conglomerado, tomando-se como base os cinco distritos sanitários existentes no município. A população correspondeu, portanto, aos adolescentes escolares matriculados no ensino médio das escolas públicas da cidade de Aracaju. 
Em cada escola, a amostra foi casual estratificada, o que implicou na divisão proporcional dos adolescentes por turnos: matutino, vespertino e noturno. Independentemente do número de séries, o universo pesquisado foi dividido por gênero.

Para definirmos a amostra, utilizamos o Epi Info versão 6.0, programado para uma freqüência esperada de $50 \%$ e aceito um intervalo de confiança de $95 \%$. Para análise, criou-se um banco de dados no módulo EPED, do programa acima referido.

Para viabilizarmos a coleta de dados, foi disponibilizado um Termo de Consentimento Livre e Informado para os adolescentes investigados e/ou responsáveis, caso dos adolescentes com idade inferior a 18 anos de idade.

A coleta de dados deu-se nos meses de julho a setembro de 1999 e foi realizada através de um questionário contendo perguntas abertas e fechadas.

Objetivando a validação do questionário, foi realizado um estudo piloto, a fim de serem verificadas as dificuldades de preenchimento e a necessidade de introdução ou supressão de perguntas.

Todos os questionários foram operacionalizados a partir do processo de codificação das variáveis.

Para análise dos dados, criou-se um banco de dados no módulo EPED do Programa EPI INFO, a partir dos módulos Analyses e Stalcalc do referido programa.

Utilizou-se o teste do Qui-quadrado para verificar a associação entre as variáveis e o teste $t$ (de student) para comparações entre as duas médias. O nível de significância para esses testes foi de alfa 0,05.

\section{RESULTADOS E DISCUSSÃO}

Aspectos demográficos e socioeconômicos

A amostra deste estudo foi constituída de 816 adolescentes do ensino médio, distribuídos proporcionalmente por escolas e turno escolar, independentemente do número total de alunos matriculados, sendo $52,3 \%$ do sexo feminino e $47,7 \%$ masculino. Do total da amostra $91 \%$ eram solteiros.

No que se refere à idade, foi detectada maior freqüência nos anos finais da adolescência, para ambos os sexos, quando $58,9 \%$ estavam concentrados na faixa etária de 18 a 19 anos.
Dos adolescentes entrevistados, 40,8\% encontravam-se trabalhando, com maior concentração para o sexo masculino, mesmo havendo número maior de mulheres na amostra.

Referente à renda individual, 79,2\% percebiam menos de um salário-mínimo, sendo a ocupação de comerciário a predominante, 34\%. Foi evidenciado que $55,1 \%$ dos adolescentes residiam com os pais, para ambos os sexos, e $25,9 \%$ viviam exclusivamente com a mãe.

Aspectos pedagógicos e comportamentais

Questionados sobre as fontes de informação sobre métodos anticoncepcionais, os adolescentes manifestaram-se conforme apresentada na Tabela 1.

Tabela 1 - Distribuição dos adolescentes por sexo, segundo fonte de informações sobre métodos anticoncepcionais

\begin{tabular}{|c|c|c|c|c|c|c|}
\hline \multirow{3}{*}{$\begin{array}{c}\text { ASPECTOS } \\
\text { COMPORTAMENTAIS } \\
\end{array}$} & \multicolumn{4}{|c|}{ SEXO } & \multirow{2}{*}{\multicolumn{2}{|c|}{ Total }} \\
\hline & \multicolumn{2}{|c|}{ Masculino } & \multicolumn{2}{|c|}{ Feminino } & & \\
\hline & $\mathrm{N}$ & $\%$ & $\mathrm{~N}$ & $\%$ & $\mathrm{~N}$ & $\%$ \\
\hline $\begin{array}{l}\text { Fontes de informações } \\
\text { sobre métodos } \\
\text { anticoncepcionais }\end{array}$ & & & & & & \\
\hline Pais & 75 & $(3,6)$ & 64 & $(3,1)$ & 139 & $(6,7)$ \\
\hline Revistas, livros, jornais & 251 & $(11,8)$ & 343 & $(16,2)$ & 594 & $(28,0)$ \\
\hline Televisão, rádio & 199 & $(9,4)$ & 184 & $(8,6)$ & 383 & $(18,0)$ \\
\hline Amigos & 180 & $(8,4)$ & 221 & $(10,4)$ & 401 & $(18,8)$ \\
\hline Namorado(a) & 70 & $(3,3)$ & 63 & $(2,9)$ & 133 & $(6,2)$ \\
\hline Professores & 94 & $(4,4)$ & 89 & $(4,2)$ & 183 & $(8,6)$ \\
\hline Profissionais de saúde & 145 & $(6,8)$ & 141 & $(6,7)$ & 286 & $(13,5)$ \\
\hline $\begin{array}{l}\text { Outros } \\
\text { TOTAL }\end{array}$ & $\begin{array}{r}02 \\
1016\end{array}$ & $\begin{array}{r}(0,1) \\
(47,8)\end{array}$ & $\begin{array}{r}03 \\
1108\end{array}$ & $\begin{array}{r}(0,1) \\
(52,2)\end{array}$ & $\begin{array}{r}05 \\
2124\end{array}$ & $\begin{array}{r}(0,2) \\
(100)\end{array}$ \\
\hline
\end{tabular}

Quando analisada exclusivamente a freqüência de informações sobre métodos anticoncepcionais, observouse que as principais fontes citadas pelos adolescentes foram: revistas, livros, e jornais, $28 \%$, seguidos de amigos $18,8 \%$, e televisão e rádio $18 \%$. Profissionais de saúde foram referidos com uma freqüência de $13,5 \%$, professores $8,6 \%$, pais $6,7 \%$, namorados $6,2 \%$ e outros $0,2 \%$.

Esses resultados apontam que, devido à falta de oportunidades em casa, o adolescente busca em revistas, livros, jornais, grupos de amigos e televisão, entre outras fontes de informação, procurando conhecer melhor sobre sexualidade e contracepção, e tentando esclarecer dúvidas existentes sobre o tema.

Relativo ao diálogo dos jovens com a família, detectamos que apenas $26,4 \%$ conversam com seus pais, ao passo que a grande maioria $(73,6 \%)$ não dialoga sobre esse tema. Esse é um fato a ser considerado, uma vez que a família juntamente com a escola, e principalmente, a primeira, são as instituições formadoras dos 
adolescentes e não se encontram discutindo efetivamente esse tema que é de suma importância para a formação pessoal desse grupo.

Outros estudos realizados ${ }^{(9-10)}$ apresentam a maior freqüência para amigos (40,9\%), e rádio e televisão (50\%), como principal fonte informativa. Reforçando os dados obtidos, quando perguntado se "conversa com os pais", apenas $6,7 \%$ dos adolescentes informaram ser essa a principal fonte de informação.

Esses achados são concordantes com pesquisas desenvolvidas que enfatizam que a educação sexual dada pelos pais é muito significante para o comportamento sexual do adolescente ${ }^{(11)}$.

Vários estudos ${ }^{(12)}$ revelam que o fato da existência de uma boa comunicação sobre sexo entre pais e filhos pode ser fator contribuinte para que a primeira relação sexual ocorra mais tardiamente.

Em relação ao conhecimento da família sobre o uso de métodos anticoncepcionais, pelos adolescentes, $61,7 \%$ afirmaram que a família não têm conhecimento, assim, apenas $11,4 \%$ do sexo feminino e $26,6 \%$ do masculino referiram que seus pais tinham conhecimento sobre o uso de anticonceptivos. O percentual de adolescentes do sexo masculino é significativamente maior que o feminino. Isso ocorre, provavelmente, pelo fato da sociedade, apesar das recentes mudanças comportamentais relativas à sexualidade, ainda aceitar mais a vida sexual ativa dos homens.

Tabela 2 - Distribuição dos adolescentes por turno escolar e aspectos pedagógicos relacionados às informações sobre métodos anticoncepcionais

\begin{tabular}{|c|c|c|c|c|c|c|c|c|}
\hline \multirow{3}{*}{$\begin{array}{c}\text { ASPECTOS } \\
\text { PEDAGÓGICOS }\end{array}$} & \multicolumn{6}{|c|}{ TURNO ESCOLAR } & \multirow{2}{*}{\multicolumn{2}{|c|}{ Total }} \\
\hline & \multicolumn{2}{|c|}{ Matutino } & \multicolumn{2}{|c|}{ Vespertino } & \multicolumn{2}{|c|}{ Noturno } & & \\
\hline & $\mathrm{N}$ & $\%$ & $\mathrm{~N}$ & $\%$ & $\mathrm{~N}$ & $\%$ & $\mathrm{~N}$ & $\%$ \\
\hline \multicolumn{9}{|l|}{$\begin{array}{l}\text { Professores } \\
\text { informam sobre } \\
\text { métodos } \\
\text { anticoncepcionais }\end{array}$} \\
\hline Sim & 55 & $(6,7)$ & 42 & $(5,1)$ & 249 & $(30,5)$ & 346 & $(42,3)$ \\
\hline Não & 41 & $(5,0)$ & 71 & $(8,7)$ & 356 & $(44,0)$ & 470 & $(57,7)$ \\
\hline TOTAL & 96 & $(11,7)$ & 113 & $(13,8)$ & 607 & $(74,5)$ & 816 & $(100)$ \\
\hline \multicolumn{9}{|l|}{$\begin{array}{l}\text { Forma de abordagem } \\
\text { do assunto }\end{array}$} \\
\hline $\begin{array}{l}\text { Superficialmente } \\
\text { Discussão }\end{array}$ & 12 & $(3,5)$ & 11 & $(3,2)$ & 93 & $(26,9)$ & 116 & $(33,6)$ \\
\hline $\begin{array}{l}\text { mostrando fatos } \\
\text { do dia-a-dia }\end{array}$ & 39 & $(11,2)$ & 22 & $(6,4)$ & 150 & $(43,4)$ & 211 & $(61,0)$ \\
\hline $\begin{array}{l}\text { Convida } \\
\text { palestrantes }\end{array}$ & 00 & $(0,0)$ & 10 & $(2,8)$ & 09 & $(2,6)$ & 19 & $(10,0)$ \\
\hline TOTAL & 51 & $(14,7)$ & 43 & $(12,4)$ & 252 & $(72,9)$ & 346 & (100) \\
\hline
\end{tabular}

$\left(X^{2} 0,05 ; 2=5,991 ; x^{2}=0,08 ; p>0,77\right)$

$\mathrm{N}=816$
No tocante aos aspectos pedagógicos, quando perguntado sobre métodos anticoncepcionais, foi verificado que $57,7 \%$ dos adolescentes não recebem informações sobre esse assunto na escola, sendo $5 \%$ no turno matutino, $8,7 \%$ vespertino e $44 \%$ do período noturno, evidenciando alto índice de adolescentes não informados sobre esse tema na escola, majoritariamente no período noturno (44\%), onde se situou também a maior concentração da amostra.

Quanto à forma de abordagem sobre o assunto, predominou a discussão em que o professor apresenta fatos do dia-a-dia (61\%), em segundo lugar a abordagem superficial $(33,6 \%)$ e palestrantes $(5,4 \%)$ (Tabela 2$)$.

Deve-se ter em conta que esses percentuais são relativos aos $42,3 \%$ que afirmaram receber informações sobre o assunto, na escola, contra os $57,7 \%$ que afirmaram não recebê-las.

A escola tem papel fundamental como suporte da família, no que concerne à educação sexual ${ }^{(12)}$, e com as mudanças na estrutura familiar a escola passa a ser um forte contexto para o desenvolvimento de uma educação sexual que provoque no adolescente um sentido de alta responsabilidade e compromisso com a própria sexualidade ${ }^{(13)}$.

A menina deveria, portanto, começar a receber orientação sexual antes da ocorrência da menarca e os meninos, além de receberem esses conhecimentos, deveriam ser sensibilizados a assumirem uma paternidade responsável de maneira a não serem surpreendidos por uma gravidez ${ }^{(14)}$.

Pode-se, pois, entender, a partir dos dados, que a Escola não tem representado, para os adolescentes, uma fonte de informação expressiva, deixando de suprir a carência dos mesmos, no tocante ao problema estudado.

Informações sobre métodos anticoncepcionais

Os dados relativos às informações dos adolescentes quanto aos métodos anticoncepcionais estão resumidos nas Tabela 3 e 4. 
Tabela 3 - Distribuição dos adolescentes por sexo e informações sobre métodos anticoncepcionais

\begin{tabular}{|c|c|c|c|c|c|c|c|c|c|c|c|c|}
\hline \multirow{2}{*}{$\begin{array}{c}\text { MÉTODOS } \\
\text { ANTICONCEPCIO } \\
\text { NAIS }\end{array}$} & \multirow{2}{*}{\multicolumn{4}{|c|}{$\begin{array}{cc}\text { Muita Informação } \\
\text { Masc. }\end{array}$}} & \multirow{2}{*}{\multicolumn{4}{|c|}{$\begin{array}{l}\text { I NFORMAÇÕES } \\
\text { ouca Inform ação } \\
\text { a s c. }\end{array}$}} & \multicolumn{4}{|c|}{$\begin{array}{r}\text { Nenhuma Informação } \\
\text { Masc. }\end{array}$} \\
\hline & & & & & & & & & $N^{M}$ & C. & & $\%$ \\
\hline Métodos naturais & & & & & & & & & & & & \\
\hline $\begin{array}{l}\text { Tabela } \\
\text { Temperatura basal }\end{array}$ & $\begin{array}{l}82 \\
06\end{array}$ & $\begin{array}{r}(10,4) \\
(0,7)\end{array}$ & $\begin{array}{c}114 \\
04\end{array}$ & $\begin{array}{r}(13,9) \\
(0.5)\end{array}$ & $\begin{array}{c}183 \\
42\end{array}$ & $\begin{array}{r}(22,3) \\
(5,1)\end{array}$ & $\begin{array}{c}259 \\
43\end{array}$ & $\begin{array}{r}(31,6) \\
(5,2)\end{array}$ & $\begin{array}{l}124 \\
341\end{array}$ & $\begin{array}{l}(15,2) \\
(41,9)\end{array}$ & $\begin{array}{r}54 \\
380\end{array}$ & $\begin{array}{r}(6,6) \\
(46,6)\end{array}$ \\
\hline $\begin{array}{l}\text { Muco cervical } \\
\text { Métodos de barreira }\end{array}$ & 04 & $(0,5)$ & 10 & $(1,2)$ & 37 & $(4,5)$ & 40 & $(4,9)$ & 348 & $(42,7)$ & 377 & $(46,2)$ \\
\hline $\begin{array}{l}\text { Condon Masculino } \\
\text { Condon Feminino }\end{array}$ & $\begin{array}{c}363 \\
56\end{array}$ & $\begin{array}{r}(44,5) \\
(6,8)\end{array}$ & $\begin{array}{r}327 \\
28\end{array}$ & $\begin{array}{r}(40,0) \\
(3,4)\end{array}$ & $\begin{array}{r}26 \\
166\end{array}$ & $\begin{array}{r}(3,2) \\
(20,3)\end{array}$ & $\begin{array}{r}95 \\
186\end{array}$ & $\begin{array}{l}(11,7) \\
(22,8)\end{array}$ & $\begin{array}{r}00 \\
167\end{array}$ & $\begin{array}{r}(00) \\
(20,5)\end{array}$ & $\begin{array}{r}05 \\
213\end{array}$ & $\begin{array}{r}(0,6) \\
(26,2)\end{array}$ \\
\hline Diafragm a & 43 & $(5,3)$ & 49 & $(6,0)$ & 198 & $(24,3)$ & 221 & $(27,0)$ & 148 & $(18,2)$ & 157 & $(19,2)$ \\
\hline $\begin{array}{l}\text { Espermicida } \\
\text { Capuz e esponja }\end{array}$ & 21 & $(2,5)$ & 16 & $(1,9)$ & 80 & $(9,8)$ & 83 & $(10,2)$ & 288 & $(35,4)$ & 328 & $(40,2)$ \\
\hline $\begin{array}{l}\text { vaginal } \\
\text { Métodos hormonais }\end{array}$ & 08 & $(0,9)$ & 06 & $(0,7)$ & 51 & $(6,2)$ & 45 & $(5,5)$ & 330 & $(40,5)$ & 376 & $(46,2)$ \\
\hline $\begin{array}{l}\text { Pílula } \\
\text { In jeção }\end{array}$ & $\begin{array}{r}173 \\
96\end{array}$ & $\begin{array}{l}(21,3) \\
(11,7)\end{array}$ & $\begin{array}{l}259 \\
145\end{array}$ & $\begin{array}{l}(31,7) \\
(17,7)\end{array}$ & $\begin{array}{r}145 \\
140\end{array}$ & $\begin{array}{l}(17,5) \\
(17,2)\end{array}$ & $\begin{array}{l}149 \\
200\end{array}$ & $\begin{array}{l}(18,3) \\
(24,6)\end{array}$ & $\begin{array}{r}71 \\
153 \\
273\end{array}$ & $\begin{array}{l}(8,8) \\
(18,8)\end{array}$ & $\begin{array}{r}19 \\
82 \\
327\end{array}$ & $\begin{array}{r}(2,4) \\
(10,0)\end{array}$ \\
\hline $\begin{array}{l}\text { Implantes } \\
\text { Outros métodos }\end{array}$ & 25 & $(3,0)$ & 16 & $(1,9)$ & 91 & $(11,2)$ & 84 & $(10,4)$ & 273 & $(33,5)$ & 327 & $(40,0)$ \\
\hline $\begin{array}{l}\text { DIU } \\
\text { Esterilização }\end{array}$ & $\begin{array}{l}26 \\
89 \\
\end{array}$ & $\begin{array}{r}(3,2) \\
(11,0) \\
\end{array}$ & $\begin{array}{l}34 \\
83\end{array}$ & $\begin{array}{r}(4,1) \\
(10,1)\end{array}$ & $\begin{array}{l}118 \\
121\end{array}$ & $\begin{array}{l}(14,5) \\
(14,9)\end{array}$ & $\begin{array}{r}143 \\
123 \\
\end{array}$ & $\begin{array}{l}(17,5) \\
(15,1)\end{array}$ & $\begin{array}{l}245 \\
179 \\
\end{array}$ & $\begin{array}{l}(30,1) \\
(21,9)\end{array}$ & $\begin{array}{l}250 \\
221\end{array}$ & $\begin{array}{l}(30,6) \\
(27,0)\end{array}$ \\
\hline
\end{tabular}

Entre os métodos naturais, o que os adolescentes afirmaram possuir mais informações foi a Tabela (Ogino Knaus), 24,3\% para ambos os sexos. A temperatura basal e o dado nenhuma informação foram respectivamente de $41,9 \%$ (masculino) e $46,6 \%$ (feminino).

Os dados referentes aos métodos de barreira apontam o condon masculino, $84,5 \%$, como o mais conhecido (ambos os sexos). Quanto aos demais métodos, capuz e esponja vaginal $(86,7 \%)$, espermicida $(75,6 \%)$, condon feminino $(46,7 \%)$, diafragma $(35,4 \%)$ apresentaram altos índices de nenhuma informação.

Os percentuais expressivos encontrados nesta pesquisa relativos ao condon masculino devem-se provavelmente às campanhas educativas que estão sendo realizadas pelo Programa DST/AIDS, no Estado de Sergipe, centradas nesse método como forma de prevenção das doenças sexualmente transmissíveis, inclusive com distribuição de preservativos em unidades escolares.

Esses achados são compatíveis com a literatura pesquisada $^{(15)}$, onde foi encontrado o percentual de $100 \%$ de adolescentes que possuíam informação sobre esse método.

No que concerne aos métodos hormonais, foi verificado que a pílula é o método que apresentou a maior concentração de informações, $53 \%$, sendo um dos mais popularmente conhecidos, talvez pelo desconhecimento dos outros métodos anticoncepcionais existentes.

Relativo ao DIU - dispositivo intra-uterino e à esterilização, foi evidenciado alto percentual para nenhuma informação em ambos os sexos; o DIU apresentou, para o sexo masculino, o percentual de $30,1 \%$, e feminino, $30,6 \%$. Já a esterilização, apesar de ser um método amplamente divulgado, 21,9\% masculino e $27 \%$ feminino afirmaram não dispor de nenhuma informação.

Esses dados demonstram que os adolescentes necessitam de mais informações sobre métodos anticoncepcionais, sendo importante que não só conheçam suas opções, como características de cada método, mas que possam também refletir sobre as questões biopsicossociais ligadas diretamente ao tema.

Tabela 4 - Distribuição dos adolescentes por sexo, segundo informações de outras práticas usadas como métodos anticoncepcionais

\begin{tabular}{|c|c|c|c|c|c|c|c|c|c|c|c|c|}
\hline \multirow{4}{*}{$\begin{array}{c}\text { OUTROS } \\
\text { MÉTODOS } \\
\text { A NTICONCEP- } \\
\text { CIONAIS }\end{array}$} & \multicolumn{12}{|c|}{ IN F O R M A ÇÕ E S } \\
\hline & \multicolumn{4}{|c|}{ Mu ita Inform a ção } & \multicolumn{4}{|c|}{ Pouc a Inform a ção } & \multicolumn{4}{|c|}{$\begin{array}{c}\text { Nen } h \text { u m a } \\
\text { Inform a çã o }\end{array}$} \\
\hline & \multicolumn{2}{|c|}{ M a s c. } & \multicolumn{2}{|c|}{ F e m. } & \multicolumn{2}{|c|}{ M a s c. } & \multicolumn{2}{|c|}{ F e m } & \multicolumn{2}{|c|}{$\mathrm{M}$ a s c } & \multicolumn{2}{|c|}{ F e m } \\
\hline & $\mathbf{N}$ & $\%$ & $\mathbf{N}$ & $\%$ & $\mathbf{N}$ & $\%$ & $\mathbf{N}$ & $\%$ & $\mathbf{N}$ & $\%$ & $\mathbf{N}$ & $\%$ \\
\hline Coito interrompido & 108 & $(13,2)$ & 104 & $(12,7)$ & 64 & $(7,8)$ & 119 & $(14,6)$ & 216 & $(26,6)$ & 205 & $(25,1)$ \\
\hline $\begin{array}{l}\text { Lavag. coca-cola } \\
\text { Aplic. creme }\end{array}$ & 18 & $(2,3)$ & 11 & $(1,3)$ & 56 & $(6,8)$ & 38 & $(4,6)$ & 315 & $(38,7)$ & 378 & $(46,3)$ \\
\hline vaginal & 45 & $(5,6)$ & 06 & $(0,7)$ & 125 & $(15,3)$ & 45 & $(5,5)$ & 219 & $(26,8)$ & 376 & $(46,1)$ \\
\hline $\begin{array}{l}\text { Lav. vaginal com } \\
\text { aspirina }\end{array}$ & 08 & $(0,9)$ & 03 & $(0.3)$ & 44 & $(5,4)$ & 26 & $(3,3)$ & 337 & $(41,4)$ & 398 & $(48,7)$ \\
\hline $\begin{array}{l}\text { Beber água com } \\
\text { sal }\end{array}$ & 23 & $(2,8)$ & 20 & $(2,5)$ & 56 & $(6,8)$ & 51 & $(6,4)$ & 310 & $(38,9)$ & 356 & $(42,6)$ \\
\hline
\end{tabular}


Quando analisadas as informações sobre métodos anticoncepcionais não convencionais, citados propositadamente no questionário, com o intuito de detectar se os adolescentes possuíam realmente informações sobre métodos anticoncepcionais, foi verificado que, apesar de um número pouco expressivo de informações sobre esses métodos, alguns adolescentes acreditavam que esses possuíam alguma eficácia, demonstrando mais uma vez a falta de informações sobre métodos anticonceptivos.

\section{CONCLUSÕES}

Considerando esses resultados, podemos então afirmar que a vida sexual dos adolescentes é uma realidade inegável, o que torna imprescindível sua conscientização e orientação, a fim de evitar gravidezes não planejadas e propiciar maior responsabilidade sobre a anticoncepção, uma vez que esse grupo necessita de informações concretas acerca do assunto.

A falta de informações sobre métodos anticoncepcionais é particularmente importante, pois o número de gravidez na adolescência vem se elevando,

\section{REFERÊNCIAS BIBLIOGRÁFICAS}

1. Sonia MMA, Paiva S. Adolescência: informações sobre anticoncepção. Rev Gauch Enfermagem 1998 janeiro; $9(1): 23-8$.

2. Cássia BR, Sônia SM. Como as mulheres relatam a participação masculina na contracepção. Rev Baiana Enfermagem 1996 abril; 9(1):53-74.

3. Organização Pan Americana de Saúde. Salud del adolescente: priodidad y estrategias nacionales y regionales. Bol Of Sanit Pan-am 1989;107(1).

4. Ministério da Saúde (BR). A saúde de adolescentes e jovens: uma metodologia de auto-aprendizagem para equipes de atenção de saúde - módulo I. Brasília (DF): Secretaria de Políticas Públicas de Saúde e Secretaria de Assistência à Saúde; 2000.

5. Fundação IBGE. Síntese dos indicadores sociais 2000. Estudos e pesquisa de informações demográficas e socioeconômica. (5). Rio de Janeiro; 2000.

6. Ministério da Saúde (BR). Secretaria Executiva de Coordenação da Saúde da Criança e Adolescente. Programa de Saúde do Adolescente: bases programáticas. $2^{a}$ ed. Brasília (DF): Ministério da Saúde; 1996.

7. Fundação IBGE. Censo Demográfico 2000: características da população e os domicílios: resultados preliminares do universo: Rio de Janeiro; 2001. trazendo muitas complicações que recairão não somente sobre os adolescentes, especialmente a mulher, bem como para a criança, a família e toda a sociedade.

A escola, como um dos principais responsáveis pela educação do indivíduo, não vem de fato assumindo seu papel, que é também participar das transformações socioculturais ligadas à questão sexual.

A família deve ser incorporada ao processo de formação dos adolescentes, pois, muitas vezes, possui informações distorcidas sobre o tema, além de apresentar dificuldades em lidar com sua própria sexualidade, devendo a escola e serviços de saúde encontrarem estratégias para atraí-las.

A escola, unidades de saúde e família devem atuar de forma integrada, de modo que o trabalho educativo encontre, na prática, o devido respaldo para transformar conhecimentos em atitudes e atitudes em comportamento, com a criação de oportunidade para que os adolescentes não só conheçam os métodos contraceptivos, mas reflitam sobre as questões biopsicossociais ligadas ao tema. Os educadores (professores, família e profissionais de saúde) poderão gerar comportamentos éticos e de respeito mútuo, bem como promover a integridade e a qualidade de vida desse grupo populacional.

8. Secretaria de Estado da Educação, Desporto e Lazer (SE). Consolidados dos estabelecimentos escolares. Serviços de Informação e Estatística. Aracaju (SE); 1997.

9. Schor N, Lopez F. Adolescência e anticoncepção. Rev Saúde Pública 1990 dezembro; 24(6):506-11.

10. Vivarta V. Mídia: quando a informação é o melhor remédio. In: Ministério da Saúde (BR). Secretaria de Políticas Públicas de Saúde. Área do Adolescente e do Jovem. A Saúde do Adolescente. Cad Juventude Saúde e Desenvolvimento 1999 agosto; 1(2):213-22.

11. Moore S, Rossenthal D. Adolescent's perceptions of friend's and parent's atitude to sex and sexual risk-talking. Commun Asppl Soc Psychol 1991; 1(4):189-200.

12. Creatsas G. Improving adolescent sexual berhavior: a tool for better fertility outcome and safe motherhood. Int $\mathrm{J}$ Ginecol Obstet 1997; 58:85-92.

13. Boruchovitch E. Fatores associados a não utilização de anticoncepcionais na adolescência. Rev Saúde Pública 1992 dezembro; 26(6):437-43.

14. Sctwarcz R, Dias AG, Fescina R. Saúde reprodutiva materna perinatal: atenção pré-natal e de parto de baixo risco. Montevidéu: CLAP-OPS/OMS; 1996.

15. Castelon $A D$, Langevim MAR. Relacion entre grado de conocimento, uso de métodos anticonceptivos y ocurrência de embarajo em adolescentes premigestas. [tese]. Santiago(Chile): Pontifícia Universidade Católica de Chile; 1993. 